\title{
Virtual Environments for Sport Analysis: Perception-Action Coupling in Handball Goalkeeping
}

\author{
Nicolas Vignais ${ }^{1}$, Benoit Bideau ${ }^{1}$, Cathy Craig $^{2}$, Sebastien Brault ${ }^{1,2}$, Franck Multon ${ }^{1,3}$ and Richard Kulpa ${ }^{1,3}$ \\ ${ }^{1}$ M2S Laboratory, UFR APS, University of Rennes 2 - ENS Cachan, Avenue Charles Tillon, 35044 Rennes, France \\ ${ }^{2}$ School of Psychology, Queen's University Belfast, N. Ireland \\ ${ }^{3}$ IRISA BUNRAKU project, Campus de Beaulieu, 35042 Rennes, France
}

\begin{abstract}
In sports science, the link between the experimental protocol and the subject's behaviour in real condition is a key issue. Virtual reality enables to examine this topic because of the reproducibility of situations and the total control of animated humanoids in situations similar to the real world. This study aimed to analyze the influence of the degree of perception-action coupling on the performance of handball goalkeepers in a virtual environment. 8 national handball goalkeepers were asked to react to the actions of a virtual handball thrower in two conditions: a perception-action uncoupled condition (defined as a judgment task) and a perception-action coupled condition (defined as a motor task). In the judgment condition, goalkeepers were asked to make a perceptual judgment with their hand in their own time after the virtual throw; in the motor task condition, goalkeepers had to react in real-time to the virtual throwing motion. Results showed that percentage of successful response was higher in the motor task condition and radial error (distance between the ball and the closest limb when trial was unsuccessful) was lower for the same condition. Implications of our findings are discussed, as well as suggestions for further research..
\end{abstract}

Index Terms-perception-action coupling, real-time, virtual reality.

\section{INTRODUCTION}

In exercise and sport science research, the link between the experimental protocol and the subject's behaviour in real conditions is an important area of research. Ecological studies consist in carrying-out experiments in real situations without interfering with the natural behaviour of the subjects. However, it generally leads to uncontrolled situations that make it difficult to identify clear relationships between causes and effects. In other words, isolating variables is almost impossible as the situations are not enough standardized. However, understanding the perception-action coupling is essential in the performance of any visuomotor task. By using our perceptual systems to pick up relevant information from the environment we can then use this information to guide our actions (e.g. catch a ball). Perception informs movements and movement informs perception (Gibson, 1979). Historically the predominant influence of an information processing approach has often created rigorous and tight

Manuscript Received on 10 November 2008

E-Mail: nicolas.vignais@univ-rennes2.fr experimental conditions. Consequently perception and action has been separated during experimental studies, especially for ball interception tasks [1, 2, 3, 4]. Thus studying perception experimentally without action can be meaningless for understanding interceptive movement.

Several studies have validated the ecological approach in the domain of coincidence timing [5, 6] and neurophysiological support for perception-action coupling has also been demonstrated [7]. Goodale and colleagues have argued that two different neural pathways are involved in visuomotor processing with the ventral stream being involved in the identification and classification of the visual stimuli (perceptual task) and the dorsal stream is more involved with the control of motor responses in response to the visual stimuli (motor task) [7, 8].

Farrow and Abernethy have studied the influence of the degree of the perception-action coupling on tennis players' anticipatory capabilities [9]. Based on video observations, novice and expert participants had to predict the direction of tennis serves in two conditions: in the judgment mode, participants verbally predicted the direction of the serves (forehand or backhand), and in the motor response mode, participants made a movement response to that which they would use to return in a game situation. These authors have shown that expert players' predictions are more accurate in the motor task condition than in the judgment condition, only if ball flight information is available. Farrow and Abernethy suggested that different perceptual processes may occur in anticipatory tasks depending on the degree of perception-action coupling. Nevertheless, using videos leaded to limited perception of the $3 \mathrm{D}$ trajectory of the ball. Moreover, the point of view of the user is not adapted to his position.

Studying the degree of perception-action coupling in real environments is very difficult because of the shortage of visual information control. For example, when studying interceptive tasks in real situations, it is not possible to obtain the same throwing kinematics of the opponent from one trial to another. This limitation could be overcame in virtual environments where the situation is numerically simulated. Hence, visual information can be controlled in a systematic manner, ensuring reproducibility between trials [10]. Moreover, the images displayed to the user can be adapted according to his own properties, such as his eyes position in space and his intraocular distance [11]. 
However, images used in virtual reality are the result of a long process that could alter the realism of the displayed scenery. Therefore, evaluating the actual feeling of the user, when participating in such kind of experiments, is a key issue. This subjective feeling of "being there" [12] is called presence [13]. In sports, the level of presence could be considered as sufficient if athlete's motions performed in the virtual environment are similar to motions performed in a real situation [14].

In a recent study, Ranganathan and Carlton analyzed the influence of the degree of perception-action coupling on baseball batting in a virtual environment [15]. These authors examined the batters' capabilities to distinguish between two kinds of pitch under two different response modes. In the judgment response mode, batters had to verbally predict the type of pitch, and in the motor task response mode, batters were asked to swing a baseball bat to try and hit a virtual ball. Contrary to findings from Farrow and Abernethy [9], results showed that batters were more precise in predicting the kind of pitch in the judgment condition than in the motor task condition. The authors explained their conflicting results by the fact that the task was more difficult for batters (swing a bat with temporal precision) and by the fact that batters had more time to make their decision in the judgment response mode, and consequently more time to process information. However, Ranganathan and Carlton have not evaluated participants' level of presence so it is not possible to know if the batters would have reacted in the virtual environment as in a real-match situation.

Conversely, Bideau and colleagues have shown that handball goalkeepers' reactions when intercepting a ball thrown by a virtual opponent were similar to those measured in real situations [16], [17], [18]. It means that the global framework is acceptable for studying such a situation in virtual reality with possible ecological extrapolations. We thus used the same framework for studying goalkeepers' abilities to estimate the final ball position in the goal mouth after a virtual throwing motion in two conditions: an uncoupled response mode where the goalkeeper was asked to manually predict the final ball position (judgment task), and a coupled response mode where the goalkeeper had to stop the ball as in a real-game situation (motor task).

\section{METHODS}

The experimentation consisted of two steps. In the first phase, we captured the motion of handball throwers and used the resulting data in a virtual environment. In this preliminary phase, we aim to obtain convenient data for animating realistic movements of a virtual thrower. In the second phase, we recorded the goalkeepers' reactions to the resulting virtual throws thanks to two methods: with and without using real-time motor actions.

\subsection{First step: Motion capture of throwers and generation of the animation in the virtual environment}

1) Participants: The participants were two top-level handball players. They were right-handed and had at least 10 years experience playing handball in the top French league at the time of the study. They gave their informed consent before participating in the experiment.

2) Apparatus: The VICON motion capture system (Oxford Metrics Ltd, Oxford, UK) was used to record kinematic data from the handball players. We used 12 infrared cameras capturing 36 reflective markers at a sampling frequency of $160 \mathrm{~Hz}$. The markers were placed at standardized anatomical landmarks [19]: sterno-clavicular joint, xiphoid process, 7 th cervical vertebra, 10th thoracic vertebra, and for both hemi-bodies, occipital and frontal bones, gleno-humeral joint, lateral humeral epicondyle, ulnar styloid process, radial styloid process, $3^{\text {rd }}$ process of the $3^{\text {rd }}$ metacarpus, anterior iliospinale, posterior iliospinale, lateral tibiale, lateral malleolus, heel, head of the $2^{\text {nd }}$ metatarsus. This marker placement enables us to reconstruct the $3 \mathrm{D}$ position and orientation of each body segment. 6 additional reflective markers were attached to the ball to capture its trajectory.

3) Procedure: After going through usual warm-up drills, we attached the reflective markers on the players. Then they were alternately asked to throw the ball 12 meters from the goal aiming for different pre-specified target zones within the goal (no goalkeeper was present). The targets were arranged so that the goal was divided into nine areas of equal size (Fig. 1). For each throw, the movement of the subject and ball were captured thanks to the reflective markers and the VICON system. Among all the trials, we selected nine movements associated with a ball reaching an area close to the centre of each zone.

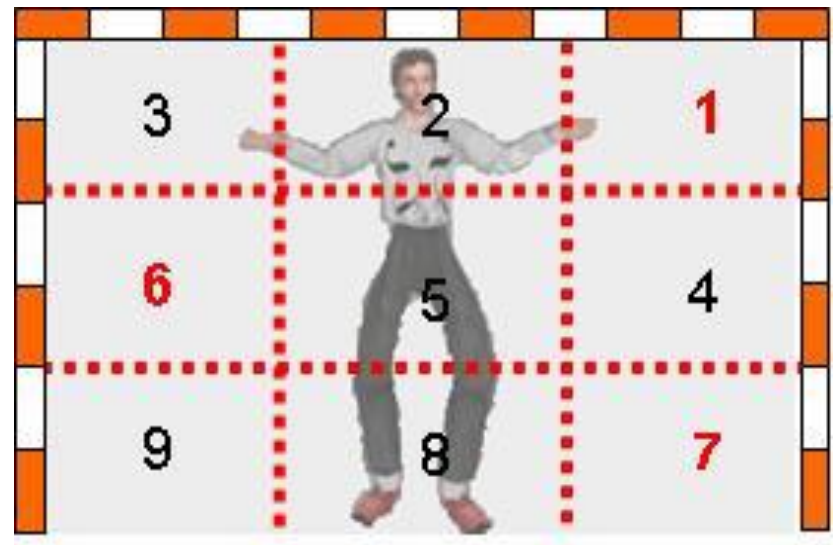

Fig. 1. Handball goal mouth divided into nine zones. Although all of the nine zones were aimed at by the thrower only zones 1,6 and 7 were used in the forthcoming analysis.

4) Creating the animation: Captured throwing data were then incorporated into the animation module Manageable Kinematic Motion (MKM, IRISA, Rennes, France) $[18,20,21]$. This module enabled us to animate the virtual handball thrower in real time. Using the recorded kinematics as a base, this programme can automatically synchronize, blend, and adapt actions to fit different morphologies and other constraints imposed by the programmer. Virtual ball trajectories for the nine zones were calculated using another software module 
that incorporates ball position and time at ball release along with the position and velocity when arriving in the goal (i.e. data obtained from the motion capture part of the study). Ball velocities were similar for all nine trajectories $\left(20.0 \pm 0.2 \mathrm{~m} \cdot \mathrm{s}^{-1}\right)$, making ball flight times almost the same for all nine zones These animation modules were driven in real time by OpenMask software (IRISA, Rennes, France) [22] (Fig. 2a). Complete animation was finally loaded in a realistic handball stadium created using 3dsmax (Autodesk Inc., San Rafael, CA, USA) (Fig. 2b). The size of the virtual stadium was scaled so that one unit in the virtual environment corresponded to $1 \mathrm{~m}$ in reality. Maintaining a realistic scale was deemed important in enhancing the feeling of presence within the virtual environment [11]. The virtual court was $20 \mathrm{~m}$ wide and $40 \mathrm{~m}$ long with a clearly marked semi-circular area $(6 \mathrm{~m}$ radius) corresponding to the goal zone. In order to enhance the feeling of presence, a real goal $(3 \mathrm{~m} \times 2 \mathrm{~m})$ was placed where it was virtually represented in the computer generated environment. Goalkeepers were encouraged to touch the real goal posts to enhance the correspondence between virtual and real distances.
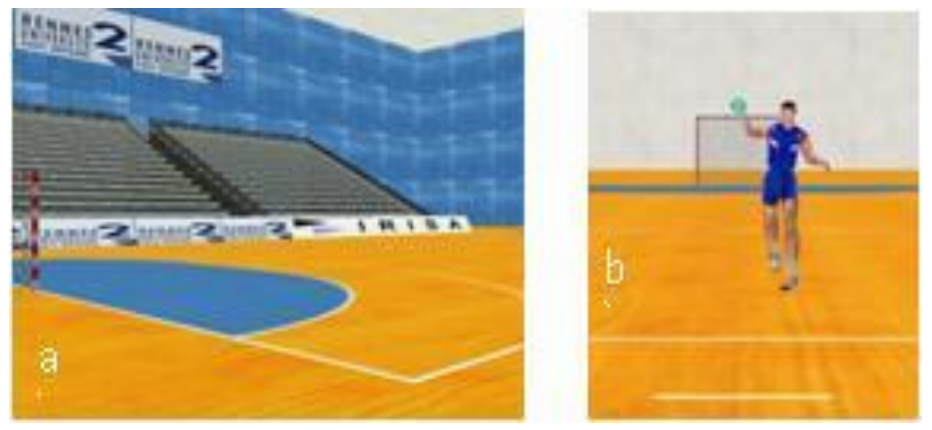

Fig. 2. Virtual handball stadium (a) and virtual handball thrower (b).

\subsection{Second step: Motion capture of goalkeepers' responses in virtual environment}

1) Participants: 8 handball goalkeepers (playing in the first division or at national level) gave their informed consent before participating in the experiment. The average age of the goalkeepers was 23.5 years $(\mathrm{SD}=$ 4.4). Mean height and mean hand length were respectively 185.6 centimetres $(\mathrm{SD}=0.06)$ and 20 centimetres $(\mathrm{SD}=1.09)$. All subjects had normal vision.

2) Apparatus: Three synchronised video projectors Barco 1208S (Barco, Courtrai, Belgium) driven by a SGI 83 Onyx2 Infinite Reality (Silicon Graphics, Sunnyvale, CA USA) were used to project the 3D sports hall environment onto a large cylindrical screen $(3.80 \mathrm{~m}$ radius, $2.38 \mathrm{~m}$ height, and $135^{\circ}$ field of vision). A set of glasses synchronised with the system enabled stereovision $(60 \mathrm{~Hz})$. The VICON motion capture system was used to record goalkeeper responses (frequency $=50 \mathrm{~Hz}$ ) and was coupled to the virtual reality display. As the two systems were linked and the goalkeeper's head was tracked, it was possible to change the goalkeeper's perspective in the virtual world in real time (delay $<20 \mathrm{~ms}$ ). In order to ensure that the size and position of the real goal posts corresponded to the virtual ones, four reflective markers were placed on the corners of the real goal indicating to the programme where the virtual goals should appear in the visual display.

3) Procedure: The goalkeeper was asked to wear a pair of glasses that enabled stereoscopic vision when looking at the display on the screen and stand inside the real goal which corresponded to the size and position of the virtual goal in the virtual environment. Two perception-action coupling conditions were then performed: a motor task condition and a judgment condition.

- Judgment task condition: in this experiment, the goalkeeper was equipped with only 11 reflective markers: 4 on their head for head tracking, 3 on their right hand, and 4 on their left hand to record final hand positions. The markers were placed on the hands in such a way that the central position of the hand could be easily calculated, with the additional marker allowing the left hand and right hand to be distinguished during analysis (see Fig. 3a). All the throws were presented in a randomised order. The ball was released when the virtual thrower was 12 meters from the goalkeeper, with the ball disappearing when it was 6 meters from the goal. All trials were cut-off $6 \mathrm{~m}$ from the goal to force the goalkeepers to make a perceptual judgment. As this was not an interception task, goalkeepers were asked to then move their hand in their own time to the position in the goal where they thought the ball would have ended up.

- Motor task condition: this experiment required the goalkeeper to get their hand to the right place at the right time to stop the virtual ball. The goalkeeper reacted to the same randomised throws displayed in the judgment condition. All trials were cut-off $6 \mathrm{~m}$ from the goal too. The goalkeeper was asked to react as if he was in a real match situation. Each goalkeeper was equipped with 36 optoelectronic markers placed on the same anatomical landmarks as in the motion capture part of the real handball throwing actions (Fig. 3b).

Before the experiment, each participant was given a training period to familiarise them with the environment and the task. During this time the participants randomly viewed a sample of throws from each of the coupling conditions (five judgment condition throws and five response condition throws). All trials in the training period were not included in the subsequent analysis.

A total of three different trajectories were presented for the different conditions (Fig. 1). They corresponded to zones 1, 6 and 7 (upper left, mid right and lower left of the goalkeeper). The three 
different ball trajectories were randomly repeated five times for each condition giving a total of 15 throws per condition. 10 other throws to different target locations in the goal were randomly included so as to create more variability in end arrival position for the goalkeeper.
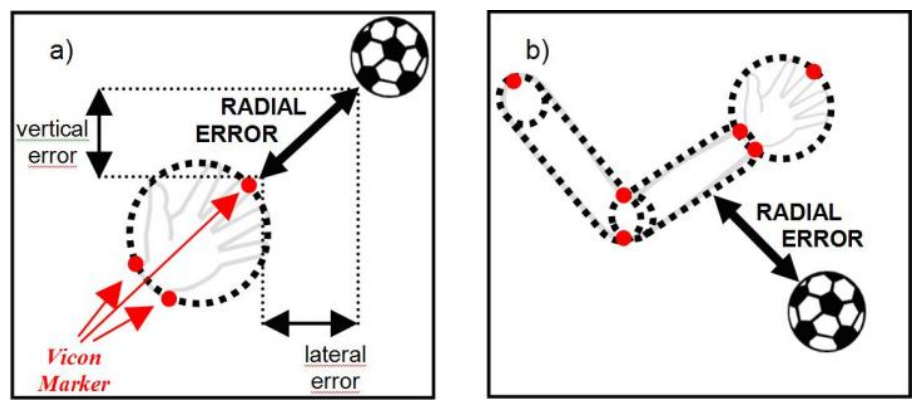

Fig. 3. Position of the optoelectronic markers, simplification of segment reconstruction and computation of the radial error in the uncoupled condition (a) and in the coupled condition (b).

4) Data analysis: In order to estimate and compare goalkeeper's perceptual and motor responses, two kinds of data analysis were performed:

- Judgment task condition: the hand and the ball were both represented by spheres (see Fig. 3a) during the process analysis. We calculated the radial error which was the difference between the final position of the hand and the virtual ball's arrival position in the goal mouth. As zero radial error equated a correct response we used this measure to calculate the percentage of successful judgments (Fig. 3a).

- Motor task condition: a detection of the collision process was used in the virtual environment by representing the goalkeeper's limbs as cylinders (trunk, arms, forearms, thighs, shanks and feet) and spheres (head and hands) from joint centre positions. This full body representation enabled us to determine if there were a collision between the virtual ball and the goalkeeper in real time (visual feedback was displayed after each throw). The collision detection process provided us with two relevant cues: percentage of successful interceptions and the goalkeeper's closest limb position (Fig. 3b).

\section{RESULTS}

1) Percentage of successful responses: The first part of the analysis looked at how the different coupling conditions influenced the participants' performance. The percentage of successful responses was also analysed. In the judgment condition, a prediction was considered successful if the end-position of the hand sphere was in contact with the end-position of the ball sphere. In the motor task condition, a parry was considered successful if the representation of the interceptive limb was in contact with the ball sphere.

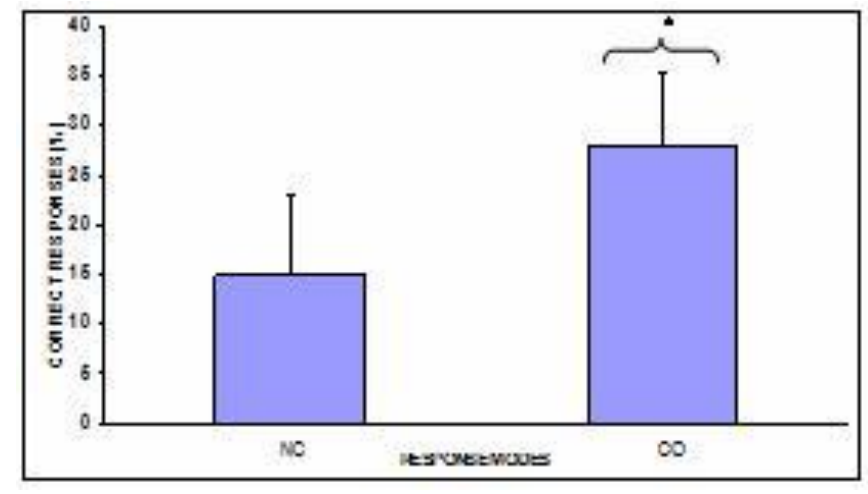

Fig. 4. Mean percentage of correct responses for all goalkeepers for each condition ( $\mathrm{NC}=$ non coupled condition; $\mathrm{CO}=$ coupled condition). The error bars represent the standard errors in the mean scores $(* \mathrm{p}<0.05)$.

As expected, the participants were most successful at performing a correct response when they were immersed in the motor task condition $(\mathrm{NC}=15 \% \pm 7.97$; $\mathrm{CO}=28.13 \% \pm 7.37$ ). A two-way repeated measures analysis of variance revealed a significant main effect for the mode of response being presented to the goalkeepers $\left(F_{(1,7)}=7.56 ; P=0.029\right)$. No significant interaction for mode of response and zone was shown $\left(F_{(2,14)}=0.38\right.$; $P=0.69$ ). A significant effect for the zone in which the ball was landed was also found $\left(F_{(2,14)}=11.29 ; P=0.001\right)$. Pairwise comparisons revealed that goalkeepers' successful responses in zone 7 were significantly higher $(P<0.01)$ than in the two other zones $(\mathrm{Z} 1=13.13 \% \pm 6.1$; $\mathrm{Z} 6=11.25 \% \pm 6 ; \mathrm{Z} 7=40.31 \% \pm 10)$. This result means that goalkeepers were more skilled at stopping virtual ball arriving in zone 7 .

2) Radial error: For each incorrect response, the goalkeeper's radial error with the correct ball position was calculated. The absolute radial errors were calculated by subtracting the position of the hand from the final position of the ball in the judgment response mode, and by subtracting the position of the closest limb from the current position of the ball in the motor response mode (Fig. 3).

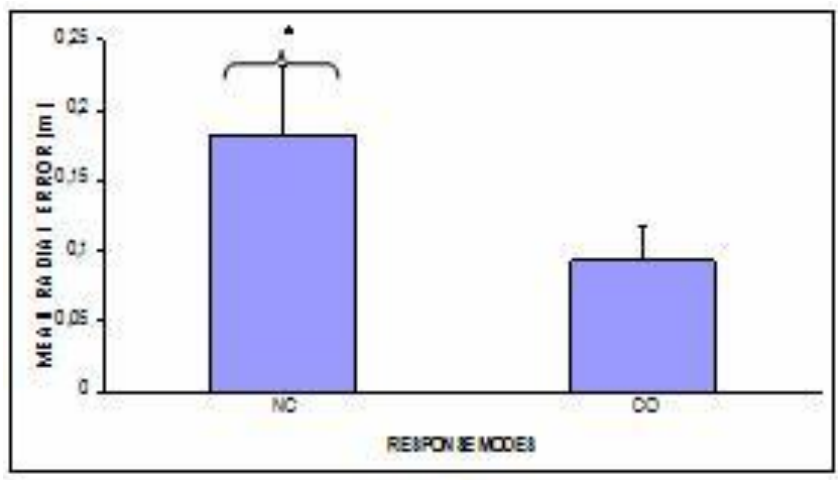

Fig. 5. Mean radial error (m) for all goalkeepers for each response mode $(\mathrm{NC}=$ non coupled condition; $\mathrm{CO}=$ coupled condition). The error bars represent the standard errors in the mean scores $(* \mathrm{p}<0.05)$.

Figure 5 illustrates the mean radial error for the judgment and motor conditions. The radial error in the 
judgment condition $(\mathrm{NC}=0.18 \mathrm{~m} \pm 0.05)$ was found to be significantly higher than in the motor condition $(\mathrm{CO}=0.09 \mathrm{~m} \pm 0.02) \quad\left(F_{(1,7)}=5.7 ; P=0.047\right)$ after a two-way repeated measures analysis of variance. No significant differences were found for the aiming zones $\left(F_{(2,14)}=0.691 ; P=0.514\right)$ and for the interaction between mode of response and zone $\left(F_{(2,11)}=0.412 ; P=\right.$ 0.672).

\section{DISCUSSION}

In the experiment reported in this paper we analyzed the visuomotor performance of handball goalkeepers under two different response modes reflecting differing perception-action coupling conditions. In the judgment condition, participants were asked to manually predict where they thought the ball would have ended up in the goal, whereas the motor task condition required goalkeepers to stop the ball as in a real-game situation.

Similarly to findings from Farrow and Abernethy [9], the results of this study showed that participants were more accurate in performing responses in the motor task condition than they were in the judgment response mode. Despite this similarity, the tasks constraints used in this study are different from those of Farrow and Abernethy's work. In our study, goalkeepers were asked to move their hand to the position where they thought the ball would have ended up in the goal although, in Farrow and Abernethy's work, tennis players just had to verbally point out the direction of the serve. This motor difference may have optimized the estimation of the final position of the ball in the goal in the judgment response mode because participants might have integrated more visual information during the displacement of the hand due to point of view adaptation and stereovision [23, 24]. This report is consistent with the ecological approach asserting that action helps perception [25]. Conversely, in the coupled response mode, participants in our study performed a more difficult task (stopping the virtual ball in real time) than those of Farrow and Abernethy's study (moving in the direction of the serve). In spite of these comments, better goalkeeping performance was found in the motor task condition when compared to the judgment condition. In order to compare these results with Farrow and Abernethy's results, a temporal occlusion technique could be used to validate the fact that a better performance in the coupled condition is found only if ball-flight information is available.

Our results considerably differ from Ranganathan and Carlton's [15] study. These authors showed that participants were significantly more accurate in performing a prediction task in the judgment response mode than in the motor response mode. They explained their conflicting results from Farrow and Abernethy's work by the fact that the task they chose was more difficult in the motor response mode and this consequently reduced prediction accuracy in this condition. Moreover they suggested the longer response times in the judgment response mode gave participants more time to process the information. Nevertheless, from our point of view, two other points would have biased Ranganathan and Carlton's results: the perception of kinematic differences and the evaluation of presence. Indeed, even if significant whole-body kinematic differences between fastballs and change-ups have been reported in college-level pitchers [26], pitching movements employed in the Ranganathan and Carlton's study resulted from a motion capture of an experienced pitcher. Consequently, kinematic differences between pitching movements may have been reduced. Besides, the framework used by these authors does not include participants' evaluation of presence. And several authors have asserted that a virtual environment must maintain a sufficient level of presence [13], [27], [28]. The virtual environment used by these authors was thereby not completely validated due to the lack of the evaluation of presence (Hendrix, 1994). In fact the level of presence reveals the degree of interaction between the participant and the environment. If this interaction does not seem "natural", which means that motor performance in the virtual environment was different than that in the virtual and in the real environments, results from such experimentation would be biased [14]. Therefore each virtual environment must be evaluated in terms of an evaluation of the level of presence, especially as it concerns sports analysis.

The present study offers support for the possible existence of special information processing modes for visual prediction and visuomotor interception. These processes depend upon the degree of coupling between perception and action. This result agrees with Milner and Goodale's findings [7]. They demonstrated the existence of two different visual processing streams for perception and action processes. However, in light of the conflicting results found in the literature, it appears that further clarification on the specificity of the task is necessary.

\section{REFERENCES}

[1] B. Abernethy, D.G. Russel, 1987. Expert-novice differences in an applied selective attention task. Journal of Psychology 9, 326-345.

[2] D.L. Wright, F. Pleasants, M., Gomez-Meza, 1990. Use of advanced visual cue sources in volleyball. Journal of Sport and Exercise Psychology 12, 406-414.

[3] A. M.,Williams, K. Davids, J.G. Williams, 1999. Visual perception and action in sport. London: E. \& F. N. Spon.

[4] K. Davids, 2002. Interceptive actions in sport: information and movement. London: Routledge.

[5] R.J. Bootsma, van Wieringen, P.C.W., 1990. Timing an attacking forehand drive in table tennis. Journal of Experimental Psychology: Human Perception and Performance 16, 21-29.

[6] C. Von Hofsten, 1987. Catching. In Heuer, H., Sanders, A.F. (Eds.), Perspectives on Perception and Action (pp.33-46). Hillsdale, NJ: Lawrence Erlbaum Associates.

[7] D.A. Milner, M.A. Goodale, 1995. The visual brain in action. Oxford: Oxford University Press.

[8] M.A. Goodale, A. Haffenden, 1998. When vision is not sight: dissociations between perception and action in human vision. In Harris, L.R., Jenkin, M. (Eds.), Vision and Action (pp.270-294). Cambridge: Cambridge University Press.

[9] D. Farrow, B. Abernethy, 2003. Do expertise and the degree of perception-action coupling affect natural anticipatory performance. Perception 32, 1127-1139.

[10] M.J. Tarr, W.H. Warren, 2002. Virtual reality in behavioural neuroscience and beyond. Nature Neuroscience 5, 1089-1092.

[11] J.E. Cutting, 1997. How the eye measures reality and virtual reality. Behavior Research Methods Instruments and Computers 29, 27-36.

[12] M.V. Sanchez-Vives, M. Slater, 2005. From presence to consciousness through virtual reality. Nature Reviews Neuroscience 6 (4), 332-339.

[13] C.M. Hendrix, 1994. Exploratory studies on the sense of presence in virtual environments as a function of visual and auditory display parameters. Phd thesis. Washington, DC, United States. 
[14] P. Zahorik, R.L. Jenison, 1998. Presence as being-in-the-world. Presence: Teleoperators and Virtual Environments 7 (1), 78-89.

[15] R. Ranganathan, L.G. Carlton, 2007. Perception-action coupling and anticipatory performance in baseball batting. Journal of Motor Behavior 39 (5), 369-380.

[16] B. Bideau, R. Kulpa, S. Menardais, L. Fradet, F. Multon, P. Delamarche, B. Arnaldi, 2003. Real handball goalkeeper vs. virtual handball thrower. Presence: Teleoperators and Virtual Environments 12 (4), 411-421.

[17] B. Bideau, F. Multon, R. Kulpa, L. Fradet, B. Arnaldi, P. Delamarche, 2004. Using virtual reality to analyse links between handball thrower kinematics and goalkeeper's reactions. Neuroscience Letters 372 (1-2) 119-122.

[18] R. Kulpa, 2005. Adaptation interactive et performante des mouvements d'humanoïde de synthétiques: aspects cinématique, cinétique et dynamique. Phd thesis. Rennes, France.

[19] D.A. Winter, 1990. The biomechanics and motor control of human movement. New York: Wiley.

[20] R. Kulpa, F. Multon, B. rnaldi, 2005. Morphology-independent representation of motions for interactive human-like animation. Computer Graphics Forum 24 (3), 343-352.

[21] F. Multon, R. Kulpa, B. Bideau, 2008. MKM: a global framework for animating humans in virtual reality applications. Presence: Teleoperators and Virtual Environments 17(1), 17-28.

[22] D. Margery, B. rnaldi, A. Chauffaut, S. Donikian, T. Duval, 2002. OpenMASK: Multi-threaded or modular animation and simulation kernel or kit: a general introduction. VRIC 2002 Proceedings, 101-110.

[23] R. Pausch, D. Proffitt, G. Williams, 1997. Quantifying immersion in virtual reality. ACM SIGGRAPH 97, 13-18.

[24] S. Lee, G.J. Kim, 2008. Effects of visual cues and sustained attention on spatial presence in virtual environments based on spatial and object distinction. Interacting with computers 20, 491-502.

[25] J.J. Gibson, 1979. The ecological approach to visual perception. Boston: Houghton Miflin.

[26] R.F. Escamilla, G.S. Fleisig, S.W. Barrentine, N. Zheng, J.R. Andrews, 1998. Kinematic comparisons of throwing different types of ball pitches. Journal of Applied Biomechanics 14, 1-23.

[27] W. Sadowski, K. Stanney, 2002. Presence in virtual environments. In Stanney, K.M. (Eds.), Handbook of Virtual Environments: Design, Implementation, and Applications (pp.791-806). Mahwah: IEA.

[28] M. Slater, A. Steed, 2000. A virtual presence counter. Presence: Teleoperators and Virtual Envionments 9 (5), 413-434.

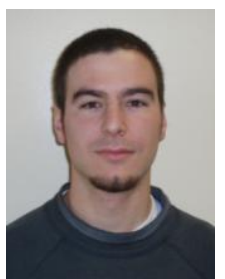

Nicolas Vignais is currently finishing his $\mathrm{PhD}$ thesis in Sports Science (biomechanics) at the M2S Laboratory (http://www.uhb.fr/labos/m2s) of the University of Rennes 2 (France). His works focuse on real-time interactions between human and virtual humanoid during a sport situation in order to understand which factors influence the level of presence. To this aim he analyses perceptive and motor task of handball goalkeepers immersed in a virtual environment under several conditions. His research involves the use of biomechanical tools such as motion capture system, animation process and virtual environment.

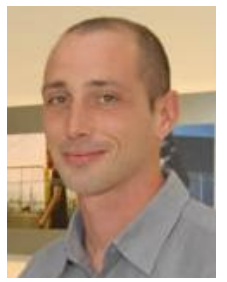

Benoit Bideau is a lecturer at the M2S laboratory, University of Rennes 2, France (http://www.uhb.fr/ abos $/ \mathrm{m} 2 \mathrm{~s}$ ). His research interests include biomechanics, perception in sport and virtual reality. Since 2000 he has been working on how to use virtual reality to study interactive behavior in different sports. One of his main goals is to provide realistic VR environments to enhance the level of presence and evaluate the influence the animation process (from motion capture to virtual human) has on improving the level of presence of immersed participants. His published studies show how handball goalkeepers react in the same way in a virtual environment as they do in a real environment. He has also shown how the MKM animation engine can be adapted to evaluate perceptual skills. He is currently developing virtual environments for different sports (rugby, handball, soccer) in order to evaluate anticipatory skill in top class athletes.

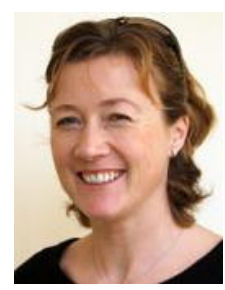

Cathy Craig is a Senior Lecturer in Visual Perception in the School of Psychology, Queen's University Belfast. Having obtained her Ph.D. at the University of Edinburgh, she went on to take up a post-doc at the Movement and Perception lab at the Sports Science Faculty at the University of Aix-Marseille 2. It was here that she first encountered virtual reality. The Movement and Perception lab was one of the first labs to use VR to study sports related skills. In a project funded by adidas she used VR to simulate curved free-kicks in soccer and investigate their effects on elite goal-keepers' anticipatory ability. Since taking up her current position at QUB, she has built an immersive interactive VR lab (https://www.qub.ac.uk/ virtualreality) where she is continuing to use VR to investigate perceptual skills in different sports.

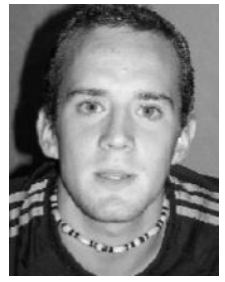

Sébastien Brault is currently a PhD Student studying Sports Science (biomechanics) at the M2S Laboratory (http://www.uhb.fr/labos $/ \mathrm{m} 2 \mathrm{~s}$ ) of the University of Rennes 2 (France) and is an international collaborator with the School of Psychology, Queen's University of Belfast. His $\mathrm{PhD}$ focuses on the application of virtual reality solutions to develop immersive sporting environments and thus, test and analyze perceptual skills. His research involves the use of biomechanical tools and perception/action analysis in order to understand the perceptual determinants of expertise in different sport's duel situations such as rugby.

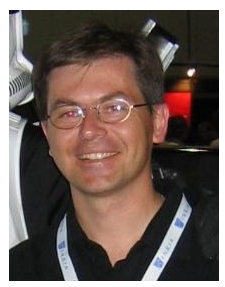

Franck Multon is Professor in University Rennes2 in France. He is performing his research in biomechanics in M2S Lab and in character simulation in Bunraku/INRIA Rennes. His research interests are biomechanics, character simulation, and interaction between real and virtual humans. He defended his PhD in 1998 in INRIA Rennes on motion control of virtual humans. Since 1999 he was Assistant Professor in University Rennes2, has defended his "authorization to supervise research" in 2006 and has been hired as full Professor in 2008. He published 20 journal papers and 23 conference papers in several domains including computer animation, robotics, virtual reality, biomechanics, neurosciences and anthropology. He is member of ACM SIGGRAPH, IEEE, and the European Society of Biomechanics. He has reviewed papers in several conferences and journals in the above domains, and was member of the international program committee of ACM SIGGRAPH SCA, CASA, IEEE-VR, GRAPP and ACM SIGGRAPH VRST.

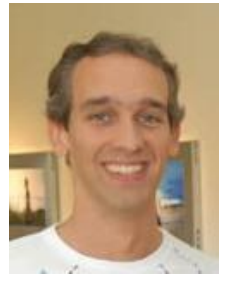

Richard Kulpa is a lecturer at the M2S laboratory, University of Rennes 2, France (http://www.uhb.fr/ labos $/ \mathrm{m} 2 \mathrm{~s})$. He is also an external collaborator of the Bunraku team of the IRISA-INRIA Rennes research institute (http://www.irisa.fr/bunraku). His research interests include biomechanics, realistic humanoids, animation and virtual reality. He worked since 1996 as a research engineer in the SIAMES team (now Bunraku) where he worked on behavioral animations of characters in informed environments in collaboration with French multimedia companies. Since 2000, in parallel to these previous works, he has integrated the LPBEM laboratory where he worked on biomechanics and its use in IK methods in order to produce fast and realistic animations. At last, he received his $\mathrm{PhD}$ in 2005 for a work that combines biomechanics and iterative inverse kinematics and kinetics solver in order to have realistic animation of hundreds of characters in real-time. He received a price at Eurographics 2005 and FMX 2008 concerning this work. By the way, he co-created the MKM animation engine that incorporates all these works. He currently uses MKM in virtual reality applications in order to evaluate performance in sports. 